\title{
Coping with cascading collective traumas in the United States
}

\author{
The year 2020 has been marked by unprecedented cascading traumas, including the COVID-19 pandemic, an \\ economic recession, race-driven social unrest and weather-related disasters. Mental health consequences of direct \\ and media-based exposure to compounding stressors may be profound. Policymakers must act to ease the burden \\ of trauma to protect public health.
}

\section{Roxane Cohen Silver, E. Alison Holman and Dana Rose Garfin}

W ith hindsight, 2020 will not easily be forgotten. Our world is in turmoil. A series of catastrophes has cascaded one to the next, and individuals across the United States have concurrently grappled with direct exposure to these events and watched them unfold, in real time, in the media. This unprecedented stressful year has both taxed the public's capacity to cope and endangered the most vulnerable groups in society.

\section{Escalating stress across America} In 2020, the COVID-19 pandemic rapidly spread from China, to Europe, the United States and globally. The pandemic overwhelmed hospitals, overtaxed healthcare workers and resulted in over 1 million deaths worldwide in 10 months, leaving families grieving in isolation. In the US alone, over 215,000 people have died in the same period. Within months, severe restrictions to limit the spread of infection left thousands of businesses closed and over 40,000,000 Americans unemployed. These crises hit low socioeconomic status and minority communities especially hard, highlighting economic and racial inequities in healthcare and essential services provision. With the pandemic and pandemic-triggered economic recession as a backdrop, Americans then faced a confluence of the current collective traumas compounded by race-based historical traumas ${ }^{1}$. Brutal killings of unarmed Black people including Ahmaud Arbery and Breonna Taylor shook the country, followed by the videotaped death of George Floyd after over almost 9 minutes with a white police officer's knee on his neck, and then by the police shooting, point-blank, seven bullets into the back of Jacob Blake. Belated recognition by whites of centuries of systemic racism in the US-primed by months of stay-at-home orders, absence of distractions, economic anxiety and easy access to gruesome videos-led directly to widespread multiracial protests, ongoing social unrest, increasing political divisiveness and violence in the streets. Simultaneously, the US has faced extreme weather events, including devastating hurricanes, record heatwaves and disastrous wildfires requiring evacuations made more complicated during an unrelenting pandemic that requires physical distancing. Together, the combination of medical, economic, racial and climate-based catastrophes highlights the need for attention to the meaning and implications of cumulative, compounding trauma exposure.

There are several characteristics of the current milieu that facilitate a perfect storm of stressors. These traumas are chronic events with an ambiguous endpoint. We do not know how bad things will get, nor when recovery can truly begin. Individuals must grapple with intense direct exposure to cascading events (for example, personal illness or loss, social isolation, economic loss, violent policing), with varying and sometimes conflicting policies dictating public response. Concurrently, these events have been broadcast in real time, as they unfolded, on traditional and social media, with individuals watching news coverage repeatedly and across multiple mediums, compounding their exposure. News has been almost entirely bad, with escalating intensity. The overlay of sensationalized media coverage in the context of repeated direct exposure to adversity is likely creating an additional crisis for public mental health.

What we know about collective trauma Decades of research on collective traumas indicates that each of these crises may independently have mental health consequences for exposed individuals, ranging from short-term anxiety to longer-term depression and post-traumatic stress disorder (PTSD) ${ }^{2}$. Although the 2003 SARS outbreak lasted less than a year, healthcare workers who cared for
SARS patients and survivors of SARS infections experienced substantial mental health difficulties ${ }^{3}$. Clinically concerning rates of mental health symptoms (anxiety, depression, PTSD) were seen in Sierra Leone among the general population exposed to the 2014 Ebola infectious disease outbreak for a year ${ }^{4}$. Population-based research before and after the mid-2000 US recession demonstrated increased risk of mental health ailments 3-4 years later among those who experienced direct consequences of the economic downturn (for example, financial, job or housing-related impacts), with low socioeconomic groups showing greater vulnerability to mental health problems ${ }^{5}$. Finally, data collected among nationally representative samples of Americans before and after police killings of unarmed Black residents demonstrated declines in mental health among Black residents in states where the killings occurred, although the state's white residents did not experience corresponding mental health deficits ${ }^{6}$.

\section{The importance of the media} In recent decades, the media landscape has changed dramatically. In addition to a round-the-clock news cycle, individuals across the world have embraced pocket-sized smartphones with easily accessible cameras that capture graphic videos of disasters and other tragedies and rapidly disseminate images widely with a click. Traditional and social media now broadcast collective traumas across the country-and globally-in record time. Yet we have only recently acknowledged that repeated indirect media-based exposure is also associated with mental and physical health ailments during infectious disease outbreaks like Ebola ${ }^{7}$, the current COVID-19 pandemic ${ }^{8}$ and following other collective traumas.

For the past two decades, using prospective, longitudinal research designs, we have examined the acute and long-term 
mental and physical health consequences of media-based exposure to collective traumas. We explored the cumulative effects of direct and indirect exposure to such events and found that real-time media exposure to multiple collective traumas (for example, the September 11, 2001 terrorist attacks; Superstorm Sandy; the Sandy Hook Elementary School massacre) was later associated with increased psychological symptomatology following the 2013 Boston Marathon bombings ${ }^{8}$. Moreover, individuals reporting several hours of combined media exposure in the days after the bombings also reported higher acute stress than individuals who were directly exposed (for example, at the Boston Marathon finish line $)^{8}$. Repeated bombing-related media exposure was also associated with ongoing worry about mass violence and traumatic stress symptoms over time. In turn, these responses predicted more media exposure following the Pulse Nightclub mass shooting in Orlando,

Florida, 3 years later ${ }^{8}$. As threats continue to emerge, these findings suggest that repeated media-based exposure to collective traumas may initiate a cycle between exposure and symptoms over time. That said, while viewing traumatic imagery may contribute to development of PTSD-like flashbacks ${ }^{9}$, we also must recognize that exposure to widely available tragic videos of police brutality, such as George Floyd's slow-motion murder under the police officer's knee, is crucial to initiate a social reckoning, such as the past-due acknowledgement of anti-Black racism in the US.

\section{Cascading collective traumas}

Multiple crises are not uncommon following natural disasters, yet research on cascading traumas is limited. For example, residents of the Biobío region of Chile experienced three rapid succession disasters in 2010: an 8.8 magnitude earthquake, a deadly tsunami and subsequent flooding, and civil unrest that resulted in days of looting. Interviews of a representative sample of over 1,000 residents at the earthquake's epicentre revealed that post-disaster distress was more strongly associated with exposure to one particular event (i.e., the tsunami) than with the number of disaster components experienced ${ }^{10}$. The tsunami appeared to be devastating because of governmental assurances that the coastal area was safe and unlikely to flood, highlighting the detrimental impact of disasters that are exacerbated by failures of trusted authorities-a lesson highly relevant to ongoing pandemic-related illnesses and deaths in the US.

Although there are limited empirical data on the consequences of compounding collective crises, more is known about the impact of cumulative exposure to lifetime adversity. Among a representative sample of over 2,000 individuals who were studied across several years, exposure to a lifetime of adversity was associated with more difficulties coping with subsequent stressors ${ }^{11}$. Indeed, having experienced more stressful life events was associated with greater distress, functional impairment and lower life satisfaction. Nonetheless, some exposure to traumatic events might serve to inoculate individuals against the distress of subsequent negative life experiences. Experiencing low (but not zero) levels of adversity may teach people what coping skills are most effective, help them engage effective support systems, promote a sense of mastery over prior crises and engender coping self-efficacy over time ${ }^{11}$. Recognition of these personal and social resources may promote resilience when one encounters the next adverse life event.

While individuals across the US may be exposed to compounding traumas both directly and via the media, mental health symptomatology in response to these exposures will vary widely. Both personal factors (for example, history of adversity, pre-existing mental health conditions, lack of economic resources) $)^{2,10,12}$ and contextual ones (for example, lack of social resources, community demographics) $)^{2,12}$ can increase vulnerability to negative psychological outcomes following collective crises. In contrast, the presence of personal and community-based resources may promote resilience and thriving in response to the stress.

\section{A call to action}

The convergence of cascading collective traumas, both historic and concurrent, raises serious questions about our future. Many unknowns remain about how individuals and communities will fare as the pandemic and economic disruption wax and wane and as worldwide cases of COVID-19 cross 40 million and deaths continue to climb. Some have warned of a possible increase in suicide and self-harm ${ }^{12}$ following chronic isolation and loneliness if quarantines are repeatedly implemented to mitigate continued infection. For people living with health disparities born of historical and racial trauma, long-term economic turmoil and loss of health insurance may exacerbate chronic health conditions, with devastating consequences.

Without intervention, might we expect people to acclimate to the unending cascade of traumas, numbing themselves to each new devastating statistic? Prior research on cumulative exposure suggests the chronicity and compounding nature of collective traumas in 2020 will likely be associated with stronger emotional responses with each new exposure, rather than habituation ${ }^{8,10,11}$. Indeed, emerging data from the US Centers for Disease Control demonstrate marked increases in adverse mental health conditions, substance use and suicidal ideation in June 2020 compared to $2019^{13}$. Therefore, how can we ensure that communities and their residents prove resilient in the face of cascading collective traumas? Prior research on communities exposed to chronic violence offers hints regarding factors that may prevent escalation of distress. A study of two Israeli communities exposed to 7 years of daily rocket fire revealed minimal levels of PTSD in the community where residents reported more community commitment, integration, strong social networks and instrumental and emotional support ${ }^{14}$. In contrast, the community where residents had high vulnerabilities, including limited education, low income and immigrant status, reported substantial symptoms of distress and PTSD. This demonstrates the value of strengthening resources distributed at both community and individual levels. Potential options include mental health support, positive coping and resilience-building activities (for example, outdoor exercise), virtual programming to reduce loneliness (particularly for those most isolated) and arts-based and life-skills based activities ${ }^{12}$.

As Black, Latinx and Indigenous communities in the US are suffering disproportionately from COVID-19, compounded by historical trauma, systemic racism and persistent poverty ${ }^{15}$, allocating additional resources to traditionally underserved and working communities of colour is critical. Underlying social inequities must be addressed to avert a mental health disaster, which will likely lead to further physical health impairments and a protracted economic and social recovery. Importantly, greater severity of exposure is likely to occur for the most vulnerable in society, adding to the burden of compounding effects. Governments must intervene to provide financial, social and emotional support to their residents, particularly those at lower socioeconomic levels. Lost pay for these individuals should be compensated, given that those lower in income will suffer the most from the economic burden of the crises. Healthcare must be provided to residents who are out of work due to the economic fallout of the pandemic and those sick with COVID19. Essential workers and minorities, who are at greater risk for problems ${ }^{13}$, must be provided the tangible (for example, personal 
protective equipment) and educational (for example, explicit safety protocols) resources needed to stay protected throughout the duration of the pandemic. Targeted outreach efforts should include additional funding for telehealth services that have demonstrated efficacy for improving mental health. This may be particularly helpful for those at highest risk for COVID-19-related complications and who must maintain maximum physical distance to protect themselves ${ }^{12}$. Finally, risk communications that promote maximum safety must be consistent at local and national levels if we are to mitigate the public health impacts of this trauma cascade.

As 2020 comes to a close while the pandemic and other tragedies continue, policymakers must make resources available to support community mental health and enact policies that directly address economic and racial inequity in the burden of these crises. In so doing, they can strengthen the social fabric and ease the mental and physical health burden of these trying times.

\section{Roxane Cohen Silver (D) 1,2,3凶,}

E. Alison Holman ${ }^{1,4}$ and Dana Rose Garfin (D) 4

${ }^{1}$ Department of Psychological Science, University of

California, Irvine, Irvine, CA, USA. ${ }^{2}$ Department of Medicine, University of California, Irvine, Irvine, CA, USA. ${ }^{3}$ Program in Public Health, University of California, Irvine, Irvine, CA, USA. ${ }^{4}$ Sue \& Bill Gross School of Nursing, University of California, Irvine, Irvine, CA, USA.

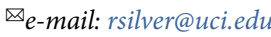

Published online: 26 October 2020

https://doi.org/10.1038/s41562-020-00981-x

\section{References}

1. Bryant-Davis, T. \& Ocampo, C. Couns. Psychol. 33, 479-500 (2005).

2. Norris, F. H. et al. Psychiatr 65, 207-239 (2002).

3. Maunder, R. G. Gen. Hosp. Psychiatry 31, 316-317 (2009).

4. Jalloh, M. F. et al. BMJ Glob. Health 3, e000471 (2018).
5. Forbes, M. K. \& Krueger, R. F. Clin. Psychol. Sci. 7, 900-913 (2019).

6. Bor, J., Venkataramani, A. S., Williams, D. R. \& Tsai, A. C. Lancet 392, 302-310 (2018)

7. Thompson, R. R., Garfin, D. R., Holman, E. A. \& Silver, R. C. Clin. Psychol. Sci. 5, 513-521 (2017).

8. Garfin, D. R., Silver, R. C. \& Holman, E. A. Health Psychol. 39, 355-357 (2020).

9. Bourne, C., Mackay, C. E. \& Holmes, E. A. Psychol. Med. 43, 1521-1532 (2013).

10. Garfin, D. R., Silver, R. C., Ugalde, F. J., Linn, H. \& Inostroza, M. J. Abnorm. Psychol. 123, 545-556 (2014).

11. Seery, M. D., Holman, E. A. \& Silver, R. C. J. Pers. Soc. Psychol. 99, 1025-1041 (2010).

12. Holmes, E. A. et al. Lancet Psychiatry 7, 547-560 (2020).

13. Czeisler, M. É. et al. MMWR Morb. Mortal. Wkly. Rep. 69, 1049-1057 (2020).

14. Gelkopf, M., Berger, R., Bleich, A. \& Silver, R. C. Soc. Sci. Med. 74, 757-766 (2012)

15. Curtice, K. \& Choo, E. Lancet 395, 1753 (2020).

Acknowledgements

Support was provided by US National Science Foundation grant SES-2026337 to R.C.S., E.A.H., and D.R.G.. D.R.G. was supported by National Institute on Minority Health and Health Disparities grant K01 MD013910.

Competing interests

The authors declare no competing interests. 